\title{
A Localization Algorithm Considering Node Movement for Wireless Sensor Network
}

\author{
Fuquan Zhang ${ }^{1 *}$, Mengmeng Shi ${ }^{1}$, Xubing Yang ${ }^{1}$, Xiao Ling ${ }^{1}$, Xinyi Tan ${ }^{1}$, \\ Demin $\mathrm{Gao}^{1}$ and Inwhee $\mathrm{Joe}^{2}$ \\ ${ }^{1}$ NanJing Forestry University, NanJing, China \\ ${ }^{2}$ Department of Electronic and Computer Engineering \\ Hanyang University, Seoul, Korea \\ zfq@njfu.edu.cn;1658366400@qq.com; xbyang@njfu.edu.cn; \\ iwjoe@hanyang.ac.kr
}

\begin{abstract}
For the application of wireless sensor networks, the perceptive information without location information is meaningless. With the popularity of mobile devices, the mobile node localization problem is attracting more and more researches. This paper, we proposed a localization algorithm. The proposed algorithm minimizes the range of sampling node by using the node's movement trajectory. This improves the efficiency of sampling and the positioning accuracy. Simulation results showed that our algorithm improves the positional accuracy.
\end{abstract}

Keywords: Localization; WSN; MCL; Algorithm

\section{Introduction}

\subsection{Background}

For the application of wireless sensor networks (WSN), the perceptive information without location information is meaningless. Generally, sensor node localization is one of factors that required in the applications, such as environment monitoring, medical health care, space search and rescue, intelligent transportation and smart home.

Now, the mechanisms used for localization can be classified into two categories: radiolocation based distance estimation and logical distance estimation. The radio-location based localization include Time Difference of Arrival (TDOA), Angle of Arrival (AOA) and Received Signal Strength Indicator (RSSI). The logical distance localization schemes include Amorphous and Approximate Point-in-Triangulation test (APIT), Distance Vector-Hop (DV-Hop). These localization schemes are designed for static scenario of WSNs [1].

In the process of localization, nodes which are needed to be localized are known as unknown nodes or common nodes. Nodes which have known their locations are called anchor node or beacon nodes.

The positioning error will increase when a node moves. Although you can use the methods of segmentation to cut time into many very small time interval, and assumed that nodes in these short time interval are relatively in static state. If the approximation wants to be a bit more precise, a shorter time interval required.

However, the location information will be transmitted over a long distance over the network, if node does not move in a very short time interval, the location information may not be transmitted. Moreover, the location information may not be precise because of the node movement. Therefore, the mobility should be considered to improve the accuracy and efficiency in positioning of node in WSNs where the node has mobility. 
It is useful to position the unknown node by studying the movement mode of anchor node in the network. MCL (Monte Carlo Localization) is a classical algorithm for position of unknown nodes when there are nodes moving in the network [10].

The remainder of this paper is organized as follows. Section 2 presents the proposed localization algorithm for mobile wireless sensor networks. Then simulation results are showed in section 3. Conclusion is in Section 4.

\section{Proposed Scheme}

\subsection{Related Works}

This work began with a thorough investigation of Monte Carlo Localization algorithm. On this basis, we proposed our scheme.

When TOA scheme estimates the distance between node A and node B, the trip time of the signal (time $\mathrm{t}$ ) that a message is transferred from/to node A and B is measured. The distance can be calculated using t multiplied by signal propagation speed. An accurate clock or synchronization is needed for trip time measurement [19].

In TDOA, two nodes $\mathrm{B}$ and $\mathrm{C}$ simultaneously send a signal to node $\mathrm{A}$. Times of arrivals $\mathrm{t} 1$ and $\mathrm{t} 2$ of signals respectively from $\mathrm{B}$ and $\mathrm{C}$ are measured by $\mathrm{A}$. The time difference of arrival td is calculated. The time difference td multiplied by signals propagation speed is mapped to the distance difference.

The TDOA of a signal can be estimated by two methods: subtracting time of arrivals TOA measurements from two anchor nodes to produce a relative time difference of arrivals TDOA or through the use methods based on cross-correlation techniques, in which the received signal at the reference sensor is correlated with the received signal at another sensor [11-15].

In AOA scheme, two nodes $\mathrm{B}$ and $\mathrm{C}$ determine the direction from which a signal from node A is coming. An imaginary line is traced from B to A, and another imaginary line is traced from $\mathrm{C}$ to $\mathrm{A}$.

The angle of arrival is defined as the angle that each of these lines makes with a line directed toward a common reference. The intersection of these two lines unambiguously determines the position of $\mathrm{A}$. Note, however, that if $\mathrm{A}, \mathrm{B}$, and $\mathrm{C}$ are all in a same line, another independent measurement is required to resolve the ambiguity.

In RSS based scheme, since a signal loses its strength as a function of distance, the distance between a sender $\mathrm{A}$ and receiver $\mathrm{B}$ can be calculated by giving the power of a transmitter and a free-space path loss model.

When three different signals are received, a receiver can determine its position in a way similar to the TOA technique. The accuracy of RSS-based scheme is generally not good due to the variation in signal strength caused by multipath fading and shadowing

The Distance Vector-hop (DV-hop) algorithm uses triangulation to localize nodes. It is assumed that there is a subset of anchor nodes among all the nodes. The position of unknown nodes is determined using the relative distance of anchor nodes [19].

In DV-hop, each anchor node advertises its position. The advertisement message is repeated from node to node. A hop count field in the advertisement message is used to control the message flooding by incremented one. Each anchor node collects the position information in advertisement messages from other anchor nodes, and constructs a vector contains the coordinates of anchor nodes.

MCLAS (Monte Carlo Localization based Anchor Node Select Model) brings neighbor nodes into reference nodes in course of sample, and select the nearest nodes which distribute symmetrically to construct sample box. In order to avoid the excessive use of bad sample, the sample's weight is weighted by angle of sample and moving direction of node which is forecasted by motion model [2]. 
MCB (Monte Carlo Localization Boxed) algorithm reduces the estimation error in location by limiting the sample box range. However, the configuration of the node ranging increases the cost of the hardware module [3].

SDANMCB (Sparse Distributed Anchor Node Monte-Carlo boxed) localization algorithm transfers node with high positioning precision to virtual anchor node to assist other nodes for localization; It adjusts sample numbers for positioning with area of sampling box and amounts of neighbour anchor node. It also adjusts weight of samples with posterior distribution of sample after filtering [22].

MMCL (Multi-hop-based Monte Carlo Localization) combines MCL and DV-Hop (Distance Vector-Hop) algorithms [4] and improve the precise of positioning without the information of the communication radius of nodes when node density is relatively low [5].

MSL (Mobile and Static Sensor Network Localization) not only takes the location from seeds into account but also uses the location from one-hop and two-hop non-seed neighbors [6].

Self-adjusting MCL localization (SA_MCL) scheme considers the characteristics of mobile sensor node. It uses interpolation simulation method to process historical location information of a node. The purpose is to get the velocity and direction of the node, thereby improving positioning accuracy. Simulation results show that SA_MCL algorithm improves positioning accuracy of a node significantly [21].

MA-MCL (Mobile-Assisted Monte Carlo Localization) relies on information of a single mobile-assisted seed that directly encountered [7]. To reduce the number of seeds, a number of mobile-assisted approaches have been proposed. Previous mobile-assisted approaches for localization require special hardware or face route selection problem. MAMCL relies on direct arriver and leaver information from a single mobile-assisted seed. It is hardware-free due to its range-free technique.

SMCL (Sequential Monte Carlo Localization) need not to form the network topologies beforehand by taking a probabilistic approach [8]. It considers the networks with sparse anchor nodes and irregular radio propagation. Unlike previously methods, SMCL takes the probabilistic approach. Because both anchors and unknown nodes can move, this character is suitable for the mobile sensor networks.

Above mentioned algorithms retain the filtering conditions, so the network has higher sensitivity to the number of anchor nodes, the time and space complexity of the algorithm are high, and the position accuracy is not ideal [9].

\subsection{Literature Review of MCL Scheme}

The algorithm contains two stages: prediction and filtration. At the prediction stage, the unknown node predicts its estimated location using reserved and the mobile information of the landmark. Assume that the velocity ranges of the node to be positioned is between 0 and Vmax. The initial position is at the o point at time t0, then, the node's location will be within the circle with radius of a Vmax, as shown in Figure 1
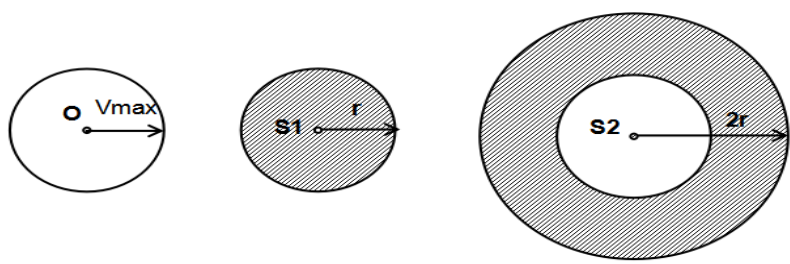

Figure 1. Constraints in Sampling Area 
At the filtration stage, the unknown node removes the inconsistent information from the estimated location. When the landmark density is low and the network communication condition is extremely irregular, MCL still can provide accurate localization. In the filtering stage, unknown node communicates and exchanges data with neighboring anchor node to find all anchor nodes that falls in the distance between 1-hop and 2-hop.

Filter the samples according the filtering condition. Filter out some samples, repeat the above steps until there are enough samples, and give the same weight to each sample location. Then it estimates the unknown node location according to the sample coordinates and the weights of each sample.

MCL does not require pre-measured location data. It can detect the position information of current neighboring node at some point. However, the sampling area will become larger with increasing of speed, this leads to the reduction in accuracy of positioning.

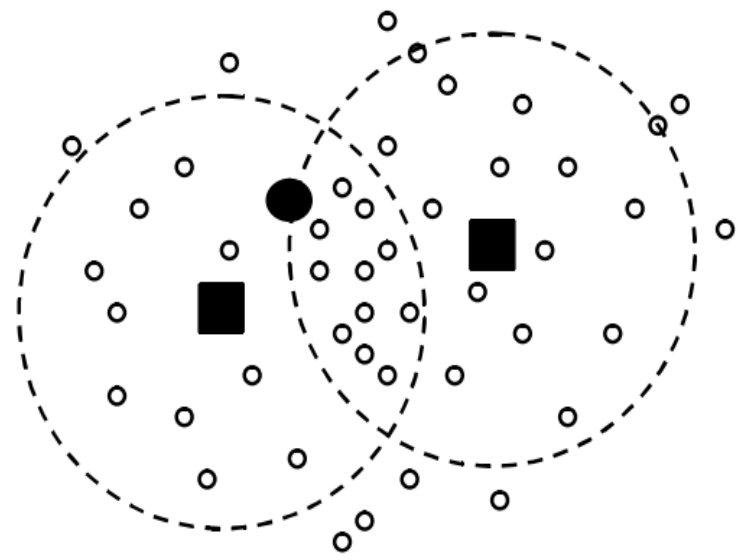

Figure 2. Sampling Area

Assumed that black square is the location where one-hop anchor node located. Black points are the unknown nodes that need to be positioning. Dashed black circle is the communication range, as shown in Figure 2. If the unknown node cannot acquire its estimated location, the prediction and filtration stage need to be implemented continually. In addition, once the filtration of the sampling fails, MCL may have to repeat above stage again and again, this leads to an infinite loop [18].

Infinite loop may lead to excessive traffic due to the exchange. A protocol should reveal very little resource in order to be widely accepted and deployed, and require low computation ability and a small amount of memory space [17].

MCL algorithm utilizes the characteristics of mobile-assisted positioning to increase localization accuracy. It is a new way to solve the mobile node localization problem in wireless sensor networks. However, when the node's velocity is increasing, the area of sampling is also increasing. This leads to the node's location calculation error increasing and positioning accuracy decreasing.

\subsection{The Proposed RMCL Scheme}

The proposed algorithm contains prediction stage and filtration stage. At the prediction stage, the unknown node predicts its estimated location using its moving direction and information of the anchor nodes [22].

If the moving direction of node is known before the sampling, the range of sampling can be restricted within the moving area. In our scheme, we try to get rid of blindness and raise the level of movement awareness. The proposed RMCL (Range Monte Carlo Localization) scheme selects the sampling area by the movement model of nodes and 
sample box. This reduces the calculation of the forecast and improves the efficiency and accuracy of positioning.

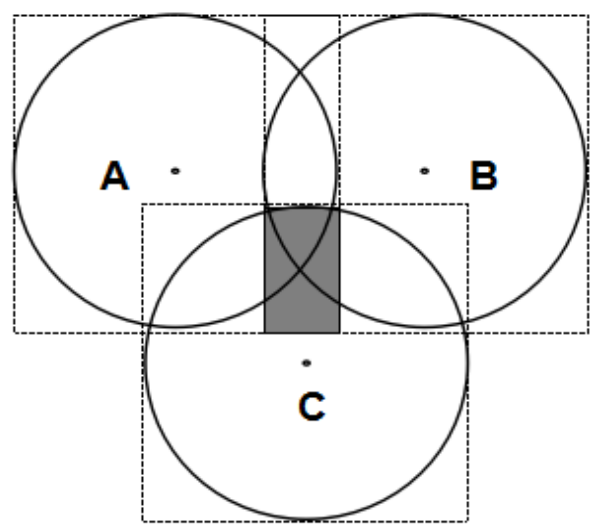

\section{Figure. 3. An Example of Sample Box}

This paper assumes that all nodes are in a two-dimensional space and the node movement is continuous without pause, so that the current location data can be used to forecast the trajectory of the node the next time to some extent. In practice, it is difficult to obtain the required and ideal parameter values based on the geographical location in MCL due to obstacles and other reasons. Since the node communication range is different with the change of the geographical position.

When calculation the sampling scope of the location, in order to calculate in convenience and solve above problem, we use a sample box for practice [3]. That is, square outside the circle are usually used to replace the circle where the node will be passed. Sample box is used to eliminate the impact and smooth the area where the communication range is irregular circle when prediction area of two anchor are intersects each other, as shown in Figure 3. In another words, the sample box uniform sampling area for each unknown node location at the present moment and conduct the sampling as the sampling area.

The location of sample nodes can be calculated by the $\left(\mathrm{X}_{\min }, \mathrm{X}_{\max }\right)$ and $\left(\mathrm{Y}_{\min }, \mathrm{Y}_{\max }\right)$ with equation 1:

$$
\begin{aligned}
& x_{\min }=\min _{j=1}^{n}\left(x_{j}-r\right) \\
& x_{\max }=\max _{j=1}^{n}\left(x_{j}+r\right) \\
& \mathrm{y}_{\min }=\min _{j=1}^{n}\left(y_{j}-r\right) \\
& \mathrm{y}_{\max }=\max _{j=1}^{n}\left(y_{j}+r\right)
\end{aligned}
$$

,where $\left(\mathrm{x}_{\mathrm{j}}, \mathrm{y}_{\mathrm{j}}\right)$ is the coordinates of the anchor node. Assumed that a node is moving with the speed $v_{x}$ along the $\mathrm{x}$-axis, and speed $v_{y}$ along the $\mathrm{y}$-axis, then the movement direction is

$$
\tan \theta=\frac{v_{y}}{v_{x}}
$$

At time $t_{0}$ the node is on the coordinate origin, the node moved with speed $\mathrm{V}_{\max }$ that can be considered as the radius $r$, then node will within the direction of motion with angle $\pm \theta$ at the next time $t_{1}$. 


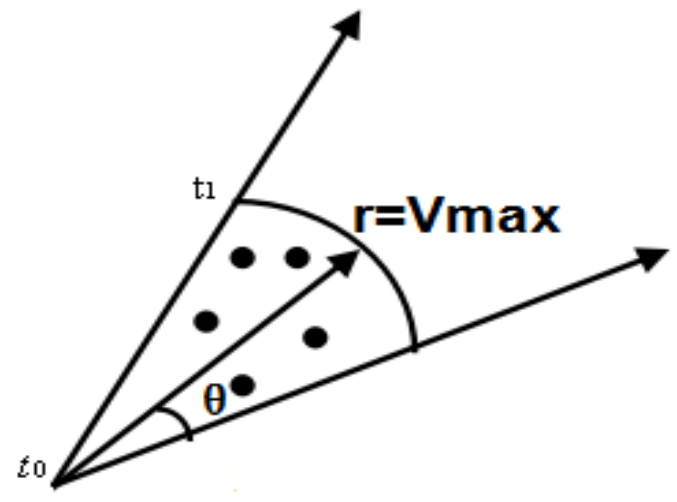

Figure 4. The Example of Movement Direction for Prediction

The overlapped area between the movement direction and the sample box is the sample area for prediction. That is, to combine the location of the motion model of each unknown node and uniformed sampling area of sample box as the sampling area for prediction. A number of points are randomly selected from the area as the predictive value of the node, as shown in Figure 4.

At the filtration stage, the filtering condition is:

$$
\text { filter }\left(r p_{i}^{n}\right)=\left\{\forall \mathrm{s} \in A_{s}, d\left(p_{t+1}^{\mathrm{i}}, s\right) \leq \mathrm{r}\right\} \cap\left\{\forall \mathrm{s} \in B_{s}, r<d\left(p_{t+1}^{\mathrm{i}}, s\right) \leq 2 \mathrm{r}\right\}
$$

In the equation, As is the single hop anchor node coordinate; Bs is the double hop anchor node coordinates; $\mathrm{r}$ is the communication range of each node; rpi is the sample coordinates.

It filters the samples in the combination of each unknown node and the observed range information of the neighboring anchor node. Filter out some samples, repeat the above steps until there are enough samples by enlarging the sampling angle $\pm \theta$. According to the sample coordinates, it is able to estimate the unknown node location $[9,10]$.

\section{Simulation Results}

The mobile model is Random Way-Point model. Sensor node is Mica2. Propagation model is Two-Ray Ground model.

\subsection{Simulation Configuration}

The simulations were done with the Two-Ray Ground propagation model, which is a radio propagation model that predicts path loss when the received signal consists of the line of sight component and multi path component formed predominately by a single ground reflected wave [16].

The Random Way Point (RWP) model is used extensively in mobile networking research and is known to have various shortcomings [17]. It is one of the most popular mobility models to evaluate mobile routing protocols, because of its simplicity and wide availability.

Our motivation for using RWP is to observe the performance of the algorithm using a well-known mobility model as well as to investigate the effects of heterogeneous mobility on the performance of the proposed algorithm.

During the simulation, the max number of anchor nodes is 40 and the number of nodes is up to 500 .

Table 1 summarizes the simulation configuration. 
Table 1. Simulation Configuration

\begin{tabular}{cc}
\hline Parameters & Values \\
\hline Propagation & Two-Ray Ground \\
mode & Modified RWP \\
Mobile mode & Mica2 \\
Nodes & up to 40 \\
Number of & \\
anchors & up to 500 \\
Number of & nodes
\end{tabular}

\subsection{Varying Number of Samples}

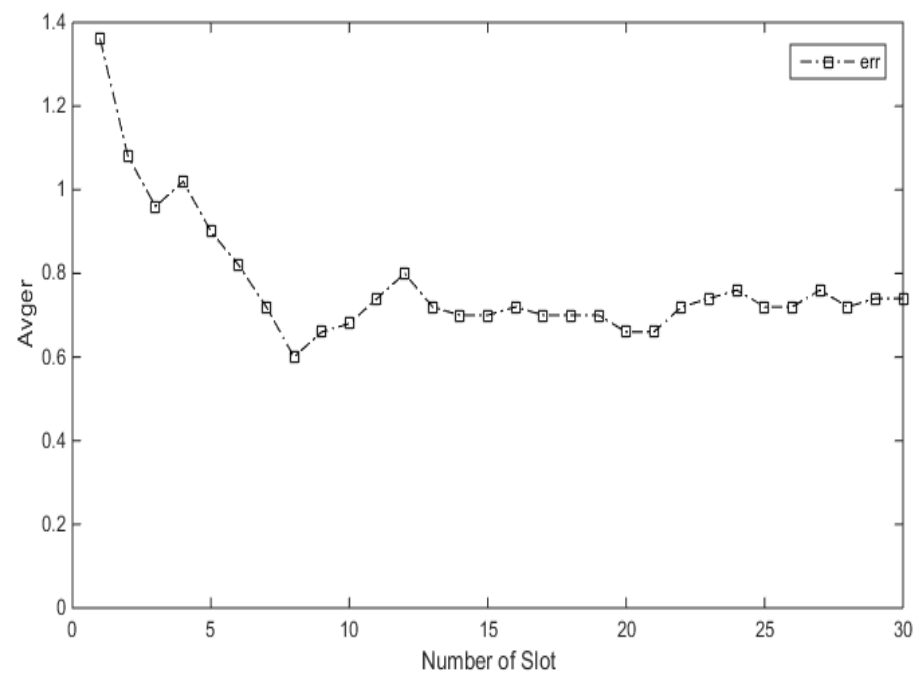

Figure 5. RMCL: The Location Error Versus Number of Samples

It is often important to take a statistically correct number of samples representing the area localized. To test for a quantifiable sample value, determine the number of samples required by simulation.

Figure 5 shows the performance with varying number of samples from 1-30. The location error decreases with the number of samples increasing. RMCL algorithm also simulates the posterior probability distribution by the discrete sampling.

Therefore, the increase in the number of samples can improve the localization accuracy. With very small sampling, the error is relatively large. The localization error is relatively stable when the number of samples is over than 10 samples.

\subsection{Varying Number of Anchor Nodes}

The proposed scheme, assumes that there are a number of anchor nodes, at the beginning, know their location by configuration or GPS. To combine the location of the motion model of each unknown node and uniformed sampling area for prediction is a method delimiting that area where a node yet to be localized is likely to be logically contained.

This may explain that increasing the number of anchor nodes is not necessary for improvement in the localization accuracy in our scheme. 
When the number of anchor nodes is increasing from 20 to 40, our scheme performs better than MCL in localization error. The location error is relatively large with small anchors for both algorithms.

The error is decreasing with the number of anchor nodes increasing initially. When the number of anchor nodes beyond a specific value, location error keeps relatively stable. MCL algorithm in this simulation is around 0.8, RMCL algorithm is around 0.5, as shown in the Figure 6.

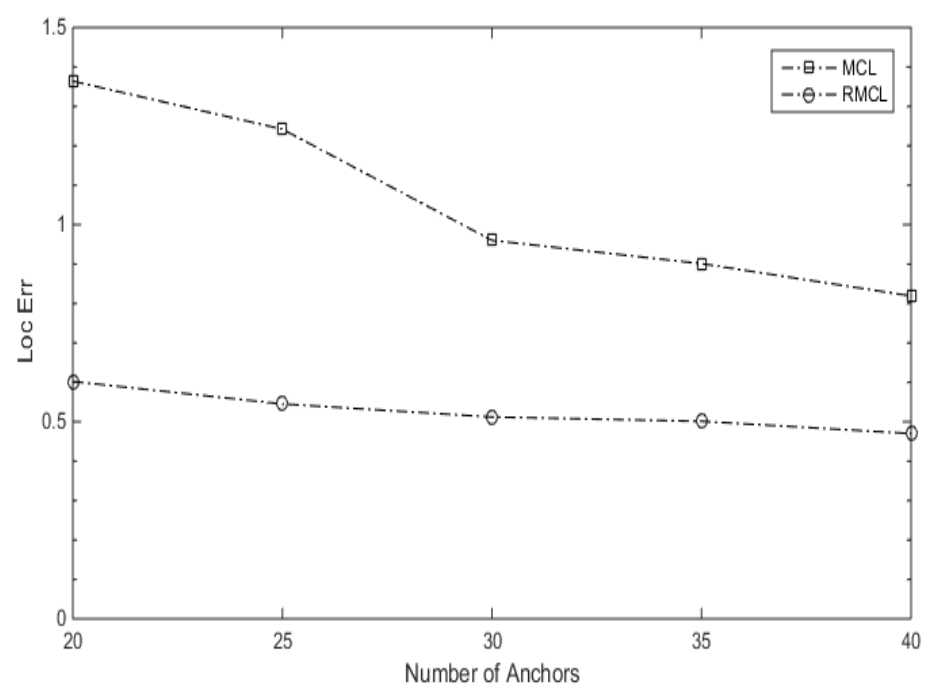

Figure 6. The Location Error Versus Number of Anchor Nodes

\subsection{Varying Number of Sensor Nodes}

The proposed scheme selects the sampling area by the movement model of nodes and sample box. As we mentioned above, since the moving direction of node is considered for sampling, the range of sampling can be restricted within the moving area.

By combining the movement direction of each unknown node and uniformed sampling area for prediction, the area of a node to be localized is decreased. Moreover, the decreasing in number of nodes for localization required less computing power.

Furthermore, the position information of anchor nodes for localization is more accurately. This can explain that increasing the number of anchor nodes is not necessary for improvement in the localization accuracy in our scheme.

The proposed scheme performs better than MCL in localization error as the number of anchor nodes increasing from 300 to 500. The location error is relatively stable and the variation is not obviously, as shown in Figure 7., when the number of sensor nodes beyond a threshold value. 


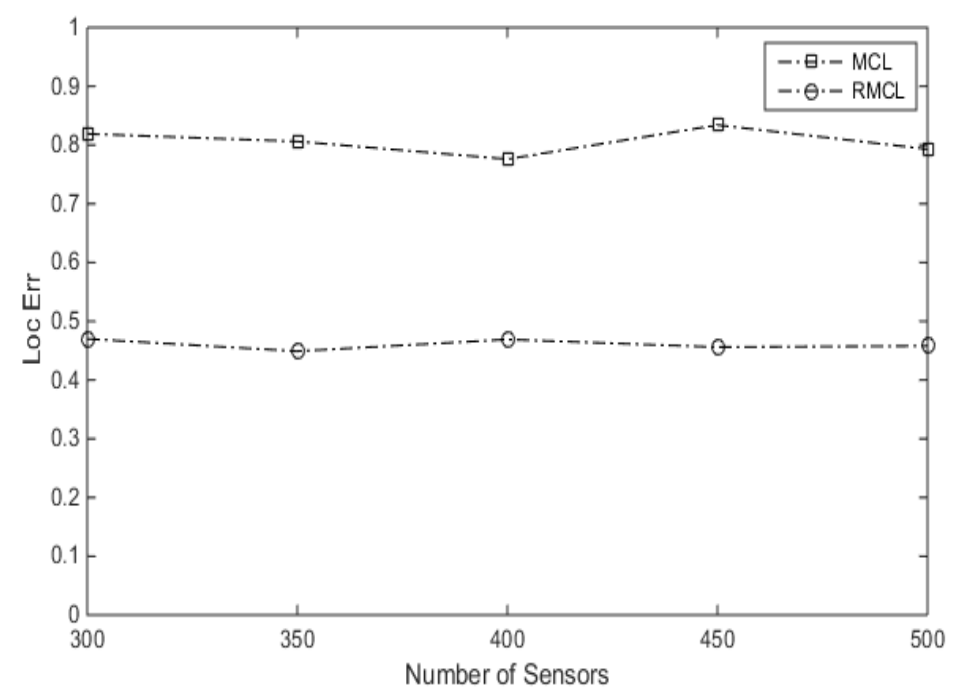

Figure 7. The Location Error Versus Number of Sensors

\section{Conclusion}

For the application of wireless sensor networks, the perceptive information without location information is meaningless. With the popularity of mobile devices, the mobile node localization problem is attracting more and more researches. This paper presented a Monte Carlo localization algorithm based scheme.

The proposed scheme reduces the sampling area by utilizing the unknown node mobility trajectory. This improves the efficiency of sampling and the positioning accuracy. Simulation results showed that our algorithm improves the positional accuracy.

We found that the localization error is relatively large and relatively stable when the number of samples is over samples threshold. Increasing the number of samples increases the localization accuracy.

Increasing the number of anchor nodes is not necessary for improvement in the localization accuracy. The location error is relatively stable and the variation is not obviously, when the number of anchor nodes beyond a threshold value.

The simulation results show that proposed algorithm improves the accuracy of positioning than related work.

\section{Acknowledgment}

This work was supported by The National Natural Science Fund, No 31670554. This work was supported by Natural Science Foundation of Jiangsu Province, Grants No BK20161527. This work was supported by TAPP:Top-notch Academic Programs Projects of Jiangsu Higher Education Institutions. This paper is a revised and expanded version of a paper entitled A Monte Carlo Based Localization Algorithm for Wireless Sensor Network presented at The 10th International Conference on Future Generation Communication and Networking, Jeju Island, Korea, 2016. The corresponding author is Fuquan Zhang. 


\section{References}

[1] G. Han, H. Xu, T. Q. Duong, J. I. Jiang, T. Hara, "Localization algorithms of Wireless Sensor Networks: a survey", Sensors, (2012).

[2] L. Min, L. Ting and X. Hua, "Localization Algorithm Based on Anchor Node Select Model for Wireless Sensor Networks", Chinese Journal of Sensors and Actuators, (2011).

[3] A. Baggio and K. Langendoen, "Monte-Carlo localization for mobile wirelesss sensor networks," Mobile Ad-Hoc and Sensor Networks, vol. 6, no. 5, (2006), pp. 718-733.

[4] D. Niculescu and B. Nath, "Ad Hoc Positioning System (APS)", Proceedings of the IEEE GLOBECOM 2001, San Antonio, (2001), pp. 2926-2931.

[5] J. Yi, S. Yang and H. Cha, "Multi-hop-based Monte Carlo localization for mobile sensor networks," in Proceedings of the 4th Annual IEEE Communications Society Conference on Sensor, Mesh and Ad Hoc Communications and Networks (SECON '07), (2007), pp. 163-171.

[6] M. Rudafshani and S. Datta, "Localization in wireless sensor networks", in Proceedings of the 6th International Conference on Information Processing in Sensor Networks (IPSN '07), (2007), pp. 51-60.

[7] G. Teng, K. Zheng, and W. Dong, "MA-MCL: mobile-assisted Monte Carlo localization for wireless sensor networks", International Journal of Distributed Sensor Networks, vol. 2011, Article ID 671814, (2011), 8 pages.

[8] W. Wang and Q. Zhu, "Sequential Monte Carlo localization in mobile sensor networks", Wireless Networks, vol. 15, no. 4, (2009), pp. 481-495.

[9] Q. Niu, T. Huan and P. Chen, "NMCT: A Novel Monte Carlo-Based Tracking Algorithm Using Potential Proximity Information”, International Journal of Distributed Sensor Networks, (2016).

[10] A. Baggio and K. Langendoen, "Monte Carlo localization for mobile wireless sensor networks", Ad Hoc Networks, vol. 6, no. 5, (2008), pp. 718-733.

[11] C. Knapp and G. C. Carter, "The generalized correlation method for estimation of time delay", Acoustics, Speech and Signal Processing, IEEE Transactions on, vol. 24, no. 4, (1976), pp. 320-7.

[12] M. Azaria and D. Hertz, "Time delay estimation by generalized cross correlation methods", Acoustics, Speech and Signal Processing, IEEE Transactions on, vol. 32, no.2, (1984), pp. 280-5.

[13] D. Hertz, "Time delay estimation by combining efficient algorithms and generalized cross-correlation methods", Acoustics, Speech and Signal Processing, IEEE Transactions on, vol. 34, no. 1, (1986), pp. 17.

[14] G. C. Carter, "Coherence and time delay estimation", Proceedings of the IEEE, vol. 75, no.2, (1987), pp. 236-55.

[15] H. I. Ahmed, P. Wei, I. Memon, Y. Du and W. Xie, "Estimation of Time Difference of Arrival (TDOA) for the Source Radiates BPSK Signal”, IJCSI International Journal of Computer Science Issues, vol. 10, iss. 3, no. 2, (2013).

[16] https://en.wikipedia.org/wiki/Two-ray_ground-reflection_model [1]

[17] A. K. , J. Ott and T. Kärkkäinen, "The ONE Simulator for DTN Protocol Evaluation”, In: 2nd International ICST Conference on Simulation Tools and Techniques, (2009).

[18] S. Jain and M. Chawla, "Survey of buffer management policies for delay tolerant networks", Journal of Engineering, (2014).

[19] Boukerche and Azzedine, "Algorithms and protocols for Wireless and Mobile Ad Hoc Networks", John Wiley \& Sons Inc, (2009).

[20] F. Zhang, M. Shi, X. Yang, X. Tan, X. Ling and D. Gao, "A Monte Carlo Based Localization Algorithm for Wireless Sensor Network", The 10th International Conference on Future Generation Communication and Networking, (2016).

[21] Y. Feihu, S. Hang, C. Lei and B. Guang-wei, "Self-adjusting Sampling Area MCL Algorithm for MobileWSNs", Computer Science, (2011).

[22] L. Hong, Z. Ziyang and W. Haopeng, "Monte-Carlo boxed localization algorithm for sparse anchor nodes environment", Transducer and Microsystem Technologies, (2014).

\section{Authors}

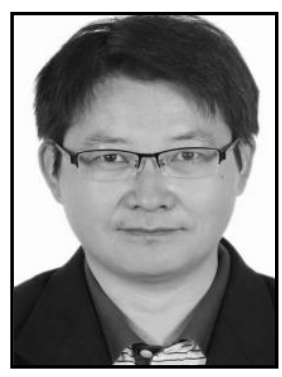

Shi Mengmeng, he is studying for her bachelor degree in College of Information Science and Technology, Nanjing Forestry University. Her research interests include wireless sensor network. 


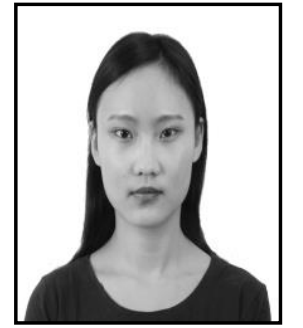

Xubing Yang, she received the B.S. degree in applied mathematics from Anhui University in 1997, MS and Ph.D. degree in computer sciences from the Nanjing University of Aeronautics and Astronautics (NUAA) in 2004 and 2008, respectively, then he joined NJFU as a Lecturer. He is an associate professor at present. His research interests include the pattern classification and neural computing.

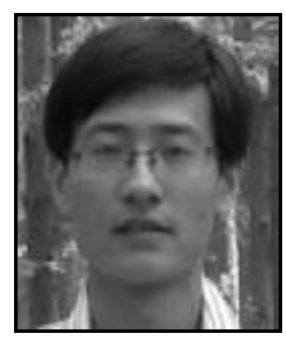

Tan Xinyi, he received an undergraduate degree in College of Information Science and Technology, Nanjing Forestry University, 2015. Her current research interests include wireless sensor network, Internet of things.

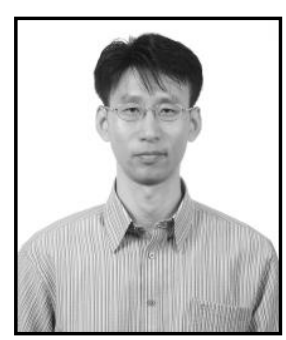

Demin Gao, he received a Ph.D. degree in 2012 from Nanjing University of Science and Technology Department of Computer Science and Engineering. In 2012, he joined in the Nanjing Forestry University as a lecturer. His research interests include routing protocols for delay tolerant, data aggregation and multiconstrained routing algorithms in wireless sensor networks and rechargeable wireless sensor networks.

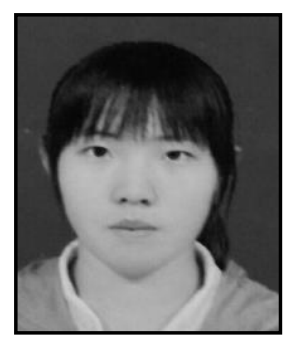

Inwhee Joe, she received B.S. and M.S. degrees in Electronics Engineering from Hanyang University, Seoul, Korea, and Ph.D. degree in Electrical and Computer Engineering from Georgia Institute of Technology, Atlanta, GA in 1998. Since 2002, he has been a faculty member in the Division of Computer Science \& Engineering at Hanyang University, Seoul, Korea. His current research interests include wireless sensor networks, 3G/4G cellular systems, mobility management, multimedia networking. 
International Journal of Future Generation Communication and Networking Vol. 10, No. 1 (2017) 